Sergio RIESE

\title{
Due nuove specie di elateridi neotropicali: Conoderus v-carinatus n. sp. e Anaissus (Anaissus) costaricanus n. sp. (Coleoptera Elateridae)
}

\begin{abstract}
Riassunto - Vengono descritte due nuove specie di Elateridae neotropicali: Conoderus v-carinatus n. sp. (Brasile, Para), caratterizzato dalla presenza di una carena a V presso la base del pronoto, e Anaissus (Anaissus) costaricanus n. sp. ( $0^{\top}+$, loc. typ: Costa Rica, prov. Alajuela, N. P. Volcán Tenorio, Mirador), affine ad A. tarsalis, ma distinto per il colore, la lunghezza delle antenne ed i caratteri edeagici.

Abstract - Two new Neotropical Elateridae species: Conoderus v-carinatus n. sp. and Anaissus (Anaissus) costaricanus n. sp. (Coleoptera Elateridae).

Two new neotropical Elateridae species are described. Conoderus v-carinatus n. sp. ( $\sigma^{\top} \&$, from Brazil, Para), belonging to the fourth Candéze's (1859) section, differs from the other species of the same section by the presence of a V-shaped carina on the pronotal base. Anaissus (Anaissus) costaricanus n. sp. ( $0^{7}$ \& , loc. typ: Costa Rica, prov. Alajuela, N. P. Volcán Tenorio, Mirador) differs from A. tarsalis in colour, longer antennae and shape of aedeagus, which is longer and slender than in A. tarsalis.
\end{abstract}

Key-words: Coleoptera, Elateridae, Conoderus, Anaissus, new species, Neotropical Region.

\section{INTRODUZIONE}

Esaminando materiale proveniente dal Carnegie Museum of natural History (CMNH) di Pittsburg, U.S.A., e dall'Instituto Nacional de Biodiversidad di Santo Domingo (INBC), Costa Rica, ho rinvenuto due specie inedite appartenenti ai generi Conoderus e Anaissus che vengono di seguito descritte.

Il materiale è depositato presso gli stessi Istituti e nella collezione dell'autore (SRGI).

La lunghezza degli esemplari è misurata dal margine anteriore della fronte all'apice delle elitre, lungo la linea mediana; la larghezza è misurata nella parte più larga del corpo.

\section{Conoderus v-carinatus n. sp. (figg. 1, 2)}

Materiale esaminato. Holotypus $\sigma^{7}$ : Para, Brazil, Acc. No., Dec. 6, 1907 Carn. Mus. Acc. 3533 (CMNH).

Paratypi (21 ess.): $3 \sigma^{x}$ e 3 ㅇ stessi dati dell'Holotypo; $90^{7}$ e 6 우: Bahia, Brazil, Dec 6, 1907, Carn. Mus. Acc. 3763 (CMNH, SRGI).

Descrizione (fig. 1). Capo giallo scuro, con una macchia discale nera di forma e dimensioni variabili, assente in alcuni esemplari. Pronoto con sei macchie: due basali, lineari, incurvate verso l'esterno fino alla carena basale, poi convergenti, estese per circa un terzo del pronoto; due laterali, oblunghe, a circa metà pronoto; due apicali, triangolari, adiacenti alla linea mediana, con l'apice del triangolo rivolto verso i lati. Prosterno giallo scuro; le suture prosternali doppie e ben distinte, nere dalla base per circa metà lunghezza. Scutello giallo scuro bordato di nero. Elitre con una macchia nera presso l'apice dello scutello, una serie di macchie nere disposte a X che si estendono sulla metà basale delle elitre e una serie di macchie apicali disposte ad ancora. La dimensione delle macchie è variabile, sia sul pronoto che sulle elitre; in alcuni esemplari le macchie sono in parte assenti.

Parte ventrale del corpo giallo scura, con due macchie lineari che partono dal mesosterno e raggiungono, restringendosi gradualmente, l'apice del settimo sternite. Antenne e zampe gialle.

Pubescenza fine, fitta, giallastra.

Capo convesso, arrotondato e ribordato al bordo distale con un leggero solco mediano e punteggiatura forte e profonda. Antenne più corte del pronoto, secondo articolo subeguale al terzo, insieme più lunghi del quarto.

Pronoto convesso, leggermente più largo che lungo, con una carena basale a forma di $\mathrm{V}$ che presenta all'apice un piccolo tubercolo; lati del protorace subparalleli per circa un terzo della loro lunghezza; angoli posteriori non divergenti, con una carena ben distinta dal bordo; superficie con punti profondi, irregolari, sul disco la distanza fra due punti adiacenti è inferiore al diametro di ciascun punto. Punteggiatura del prosterno più distanziata di quella del pronoto, apofisi prosternale diritta.

Scutello trapezoidale, arrotondato all'apice, granuloso.

Elitre ristrette subito dopo la base, tronca- 
te all'apice, ciascuna con una piccola spina apicale all'angolo interno della troncatura; strie ben incise dalla base all'apice, interstrie convesse, granulose alla base, con punteggiatura fine e rada.

Lamella del quarto articolo dei tarsi piccolissima, quasi invisibile.

Dimensioni: lunghezza 4-5,5 $\mathrm{mm}$, larghezza 1,2-1,5 mm.

Edeago dell'holotypus come nella fig. 2.

Derivatio nominis. Il nome deriva dalla forma della carena presente sul pronoto.

Note comparative. $C$. v-carinatus $\mathrm{n}$. sp. è affine alle specie collocate nella "sezione quattro" di Candèze (1859) (sub Monocrepidius), e successivamente trattate da Golbach (1987) che fornisce una chiave dicotomica per la loro identificazione.

Il gruppo è caratterizzato da piccole dimen- sioni corporee, dagli articoli 2 e 3 delle antenne subeguali, presi assieme più lunghi del quarto, e dalla lamella del quarto articolo dei tarsi molto piccola e poco visibile. Da tutti i taxa di questo gruppo (C.auritus (Herbst, 1806), C. bellus (Say, 1824), C. pictus (Candèze, 1859), C. mexicanus (Champion, 1895), C. parvulus (Champion, 1895), C. sexpustulatus (Champion, 1895), C. backeri (Hyslop, 1916), C. argentinus Golbach, 1987) C. v-carinatus n. sp. si distingue principalmente per la presenza di una carena basale a forma di V sul pronoto.

Anaissus (Anaissus) costaricanus n. sp. (figg. 3-4)

Materiale esaminato. Holotypus $\sigma^{\pi}$ - Costa Rica, Prov. Alajuela, Upala, P. N. Volcán Tenorio, Alb. Heliconias, Mirador, Send. Lag. Danto, 700 m, 21-27 Feb. 2001. G. Rodriguez. Luces. L_N_422423_298575*61619 INB0003130889 INBIOCRI Costa Rica
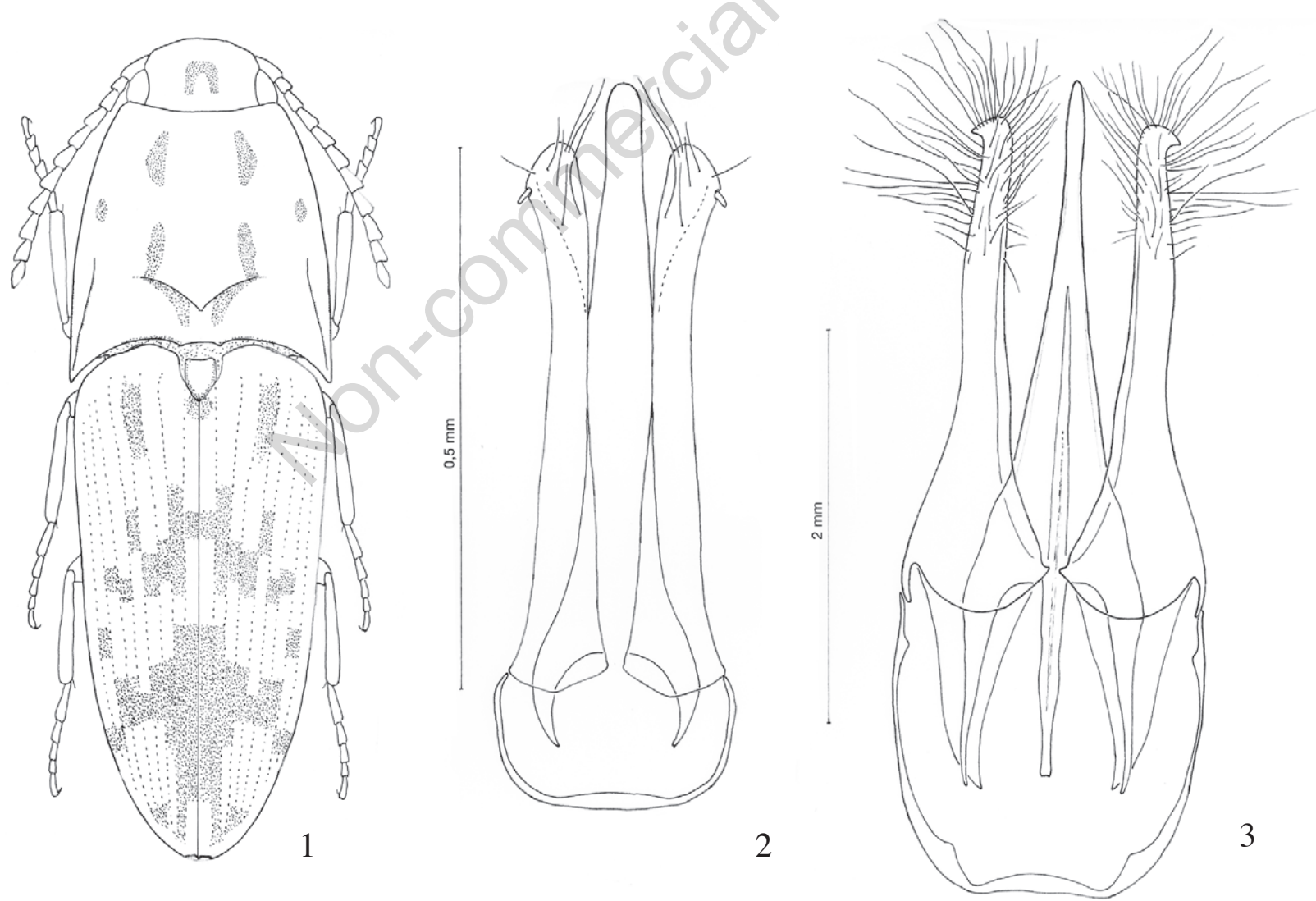

Fig. 1: Habitus di Conoderus v-carinatus n. sp., holotypus. Figg. 2-3: Edeagi degli holotypi in visione dorsale: 2 - Conoderus v-carinatus n. sp.; 3 Anaissus costaricanus n. sp. 
PARATYPI (33 ess.): $2 \sigma^{\pi}$ stessi dati dell'holotypus, numero repertorio INBC INB0003130887 e INB0003130888, 1 $0^{7}$ Costa Rica, Prov. Alajuela, Upala, P. N. Volcán Tenorio, Albergue Heliconias 700 m Abr. 2000 G. Rodriguez Manual. L_N_423800 $299800 * 58125$ INB0003172471; $1 \sigma^{\pi}$ Costa Rica, Prov. Alajuela, Upala, Bijagua, P.N. Volcán Tenorio, Albergue Heliconias, 700m Abr. 2000. G. Rodriguez. Manual. L_N_423800_299800 *58125 INB000172470; 1 ᄋ Costa Rica, Prov. Alajuela, Upala, Alb. Heliconias, Send Heliconias, Cañon. 700m 30 Ago. 2000. A. Lopéz. Luz ambiente acquatica L_N 422600 $299100 * 58508$ INBOOO3302168; 1 O Costa Rica, Prov. Alajuela, Upala, P.N. Volcán Tenorio, Alb. Heliconias, S. Heliconias 700m 24 Abr. 2001. A. López. Luz ambiente L_N_422600_299100_*62032 - INB0003173127 “Anaissus tarsalis $(\mathrm{Cd})$ det. P.J. Johnson 02; $1 \sigma^{\top}$ Costa Rica,

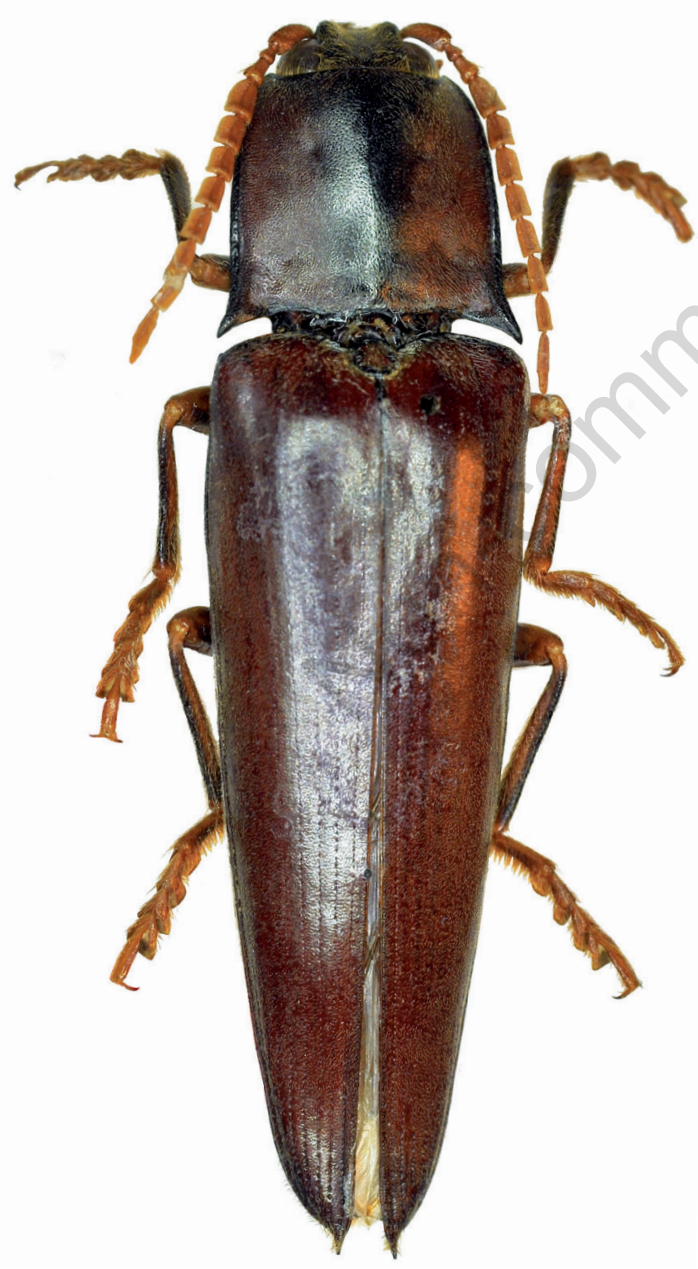

Fig. 4: Habitus di Anaissus costaricanus n. sp., holotypus.
Prov. Alajuela, Upala, P.N. Volcán Tenorio. Albergue Heliconias, Send. Heliconias, 700m 21 Ago. 2001 A. López, T. de Luz L_N_299100_422600*64798 INB0003379043; 1 을 Costa Rica, Prov. Alajuela. P.N. Volcán Tenorio, Sendero Heliconias, 700m 23-28 Ago. 2002, A. López. Tp. de Luz. L N 299100422600 *72155 - INB0003553590; 1 \& Costa Rica, Prov. Alajuela. P.N. Volcán Tenorio. Valle Rio Roble, Palmital abajo la caliza, 800-1000m 24 Jun. 2006 - J. A. Azofeifa. Tp. Luz L N 297100426600 *86653 - INB0004022979; 1 ㅇ Costa Rica, Prov. Alajuela P.N. Volcán Tenorio. Estacion Pilon, 1,5 SO c. Carmela, 700-800 m 14 Feb. 2007. J.A. Azofeifa - Libre L N 298212-427913 *91111 INB0004075981; 1 O' Costa Rica, Prov. Alajuela, A.C.A. San Ramón, Est R. B. Alberto Brenes, R. Lorencito, 850m 19-26 Mar. 1999. A. Azufeifa. Manual (red. libre) L_N_245500_470800 *52390 INB0003035058; 1 ㅇ Costa Rica, Prov. Alajuela, R:B: San Ramón. 800m Abr. 1997. G. Carballo, N. Flores. De Luz L_N_245100_472100*46306 INBIO CRI002564180; 1 \& Costa Rica, Prov. Alajuela. Sector Colonia Palmareña, $9 \mathrm{Km}$ SO de Bajo Rodriguez, $700 \mathrm{~m}$ Mar. 1997. G. Carballo. L_N_245900 $475900 * 45517$ INBIO CRI002537351; 1 ㅇ Estac. Pitilla, 700m 9km S Santa Cecilia; Guanac Pr. 27 Jan- 4 Feb 1989 GNP Biod. Sur. 330200.380200 INBIO CRI000105700, 1 우 Estac. Pitilla, 700m 9 km S Santa Cecilia, Mar 1989 - GNP Biodiversity Survey 330200.380200 INBIO CRI000024768; 3 O" e 1 오 Estac. Titilla,700m 9km S Santa Cecilia, Guanacaste Costa Rica, Mar 1990 - P.Rios, C. Moraga \& R.Blanco. 330200-38200 INBIO CRI000195704, CRI000195709, CRI000195930, CRI000176902; 1 O' Est Pitilla,700m 9km S Sta Cecilia, Prov. Guan. Costa Rica, P. Rios, Abr. 1990 L-N-330200.380200 INBIO CRI000615273 - Anaissus tarsalis Candéze det. P.J. Johnson '95; $10^{7}$ Est. Pitilla, 700m 9km S Sta Cecilia, Prov.Guan. Costa Rica, C. Moraga \& P. Rios Mar. 1991 L-N-330200.380200 INBIO CRI000341835; 1 ○ Est Pitilla, 700m 9km S Sta Cecilia, P.N. Guanacaste, Prov. Guanacaste. Costa Rica, S. Rojas 31 mar - 29 abr 1992 L-N 330200.380200 INBIO CRI000740674; 1 ○ Est Pitilla, 700m, 9 km S Sta Cecilia, P.N. Guanacaste, Prov. Guanacaste, Costa Rica, K. Taylor 31 mar- 29 abr 1992 L-N 330200.380200 INBIO CRI000523676; $10^{7}$ Est Pitilla. 700m 9 km S Sta. Cecilia, P.N. Guanacaste, Prov.Guan. Costa Rica 19 May - 3 Jun 1993 C. Moraga L-N-330200.380200 INBIO CRI001343092; 1 ○ Est Pitilla, 9 km S Santa Cecilia, P.N. Guanacaste, Prov. Guana. Costa Rica 700m 1017 Feb. 1994 P. Rios L N 330200-380200*2634 INBIO CRI001961484; 1 O Estación Pitilla, 9 km S Sta Cecilia, Prov. Guana. Costa Rica, 700 m Abr. 1994. C. Morata L N 330200-380200*2841 INBIO CRI001789982; $1 \sigma^{\top}$ Estación Pitilla $9 \mathrm{~km} \mathrm{~S}$ da Santa Cecilia, Prov. Guana. Costa Rica 700 m 4-23 Abr. 1995 E. Alfaro, L_N_329950-380450*4602 INBIO CRI002334900; 1 
$0^{7}$ Fca Campana, 5 km NW Dos Rios, Alajuela pr. Costa Rica, 750 m 21 Mar 1985, D. H. Janzen \& W. Hallwachs INBIO CRI002610768; 1 ○" Est El Celbo, Braulio Carrillo, N.P. 400-600 m, Heredia, Costa Rica Apr 1990 C. Chaves 527700-256500 INBIO CRI000210857; $1 \sigma^{7}$ R. San Lorencito, 900 m, R. F. San Ramón, 5 km $\mathrm{N}$ de Colonia Palmareña,Prov. Alaju. Costa Rica 1318 Jun 1993. I- Curso Scarabeidae L-N-244500,470700 INBIO CRI001365234; 1 ○ Sector Colonia Palmareña, Ribera del Rio San Lorenzo, Prov. Alaju, Costa Rica, 700 m, 22 Mar. 1995. F. Mejìa, G. Carballo, M. Reyes L N $245900475900 * 5453$ INBIO CRI002319711; 1 $\mathrm{O}^{\overline{-}}$ Sector Colonia Palmarena, $9 \mathrm{~km}$ SO de Bajo Rodriguez, Alajuela, Costa Rica, 100m Mar 1996 G. Carballo L_N_245900_475900*7198 INBIO CRI002450205; 1 $\sigma^{7}$ Costa Rica: Prov. Heredia 10km SE La Virgen, 450$550 \mathrm{~m}, 10^{\circ} 20^{\prime} \mathrm{N} 84^{\circ} 05^{\prime} \mathrm{W} 21 \mathrm{Feb} 2003$ INBio- OETALAS transect-21 Febrero 2003 Transect 05/LM/00/005 INBIO0003241528. (INBC, SRGI).

DesCrizIONe. Colore generale castano chiaro; protorace dorsalmente con banda centrale e carene laterali nere, inferiormente nero con episterni castano scuro; angoli basali esterni delle elitre e scutello bordati di nero. Antenne e palpi giallo arancio, zampe gialle, femori e tibie in parte oscurati, penultimo sternite con breve linea gialla apicale. Pubescenza corta, fitta.

Capo depresso centralmente, con punti forti, profondi, irregolari, più forti e radi ai lati del capo, più piccoli e fitti al centro e all'apice; pubescenza dorata. Primi tre articoli delle antenne con punti radi e profondi, di aspetto lucido, il secondo lungo circa la metà del terzo, $i$ tre insieme così lunghi come il quarto, gli altri antennomeri con punti fini e fitti, di colore opaco e con una linea mediana leggermente più scura su tutta la lunghezza; le antenne superano la base del pronoto di circa un articolo nei maschi, raggiungono appena la base del pronoto nelle femmine.

Pronoto poco convesso, leggermente più largo che lungo, a lati subparalleli, ristretti all'apice; bordo anteriore leggermente arcuato verso l'esterno, con una serie di peli gialli; disco con punti larghi e poco profondi, la distanza fra due punti ai lati del pronto è inferiore al diametro di ciascun punto; i punti sono più profondi e distanziati sulla zona centrale del pronoto; superficie microreticolata, più fittamente ai lati, di aspetto opaco e con quattro leggere depressioni, due basali oblique, due discali lineari e subparallele ai lati. Angoli posteriori divergenti, con carena breve e poco visibile.

Prosterno lucido; al centro con punti forti, profondi e radi; ai lati, alla base e all'apice con punti più piccoli e ravvicinati. Episterni con punteggiatura simile a quella del pronoto.

Scutello submitrale, arrotondato all'apice, leggermente convesso, rilevato alla base e a metà della lunghezza, con punti larghi, fitti, poco profondi.

Metasterno e sterniti con punteggiatura fine e rada; ultimo sternite all'apice con punti forti, profondi e irregolari.

Femmine con colorazione più scura e con antenne più brevi dei maschi; l'ultimo sternite dentellato a circa metà della lunghezza.

Elitre a lati subparalleli per circa un quarto della loro lunghezza, quindi convergenti verso l'apice; angoli apicali divergenti, spiniformi. Strie elitrali punteggiate dalla base fino a circa metà della loro lunghezza, poi leggermente incise, con punti progressivamente più grossi e forti. Interstrie piatte, con punti fini, radi e poco profondi, microreticolate.

Dimensioni: lunghezza $33-37 \mathrm{~mm}\left(\sigma^{7} \sigma^{7}\right), 41$ $\mathrm{mm}\left(\right.$ 우 ); larghezza 8,5-9,5 $\mathrm{mm}\left(\sigma^{7} \sigma^{7}\right), 10,5 \mathrm{~mm}$ (우 우).

Edeago dell'holotypus come nella fig. 3.

Derivatio nominis. Dal Costa Rica, paese da cui provengono gli esemplari esaminati.

Note comparative. $A$. costaricanus $\mathrm{n}$. sp. è molto simile ad $A$. tarsalis Candèze, 1857; se ne distingue principalmente per la maggior lunghezza e differente colorazione delle antenne e per la forma dell'edeago: corto e largo in $A$. tarsalis, sottile e allungato in A. costaricanus $\mathrm{n}$. $\mathrm{sp}$.

Nota. Il genere Anaissus Candèze, 1857, è stato oggetto di una recente pubblicazione (Riese, 2007), nella quale viene descritto $A$. chassaini Riese, 2007 di Panama, finora considerata la specie a distribuzione più occidentale del genere. A. costaricanus $\mathrm{n}$. sp. amplia ulteriormente verso ovest la distribuzione conosciuta per questo genere.

RINGRAZIAMENTI.

Ringrazio i Dr Robert L. Davidson (CMNH), Dr Angel Solis e Dr Alvaro Herrera (INBC) per avermi affidato il materiale in studio, e gli amici Ivo Gudenzi per i disegni e Gianni Tognon per la foto. 


\section{BiBLIOGRAFIA}

CAndèze E., 1859 - Monographie des Elaterides - 2. Mémoires de la Société royale des Sciences de Liège, 17, 534 pp.

Golbach R., 1987 - Conoderus argentinus n. sp. (Col. Elat.) y comentarios sobre especies vecinas. Acta Zoologica Lilloana, 39: $17-21$.

RiESE S., 2007 - Contributo alla conoscenza del genere neotropicale Anaissus con descrizione di sei nuove specie (Coleoptera Elateridae). Bollettino della Società entomologica italiana, 139: 91-100.

Indirizzo dell'autore:

S. Riese, corso Sardegna 46/11d, I-16142 Genova GE, Italia. sergio.riese@libero.it 